\title{
Paraplegia due to Extramedullary Hematopoietic Tissue Compression of Thoracic Thecal Sac in a Patient with Thalassemia Treated Successfully with Surgical Decompression: A Case Report
}

\author{
Ashish Tiwari ${ }^{1}$ Vineesh K. Varghese ${ }^{1}$ Ashis Kumar Chand ${ }^{1} \quad$ Manmeet Singh Chhabra ${ }^{1}$ \\ ${ }^{1}$ Department of Neurosurgery, St. John's Medical College and \\ Hospital, Bengaluru, Karnataka, India \\ Indian J Neurosurg 2016;5:31-33.

\begin{abstract}
Address for correspondence Ashish Tiwari, MS, Department of Neurosurgery, St. John's Medical College and Hospital, Bengaluru, Karnataka 560034, India (e-mail: drashishtiwari1978@gmail.com).
\end{abstract}

\begin{abstract}
Keywords

- extramedullary hematopoietic

- decompressive laminectomy

- thalassemia major

Spinal cord compression due to extramedullary hematopoiesis (EMH) is a rare complication of thalassemia and generally presents as paraparesis with sensory impairment. Complete paraplegia is extremely rare in $\mathrm{EMH}$ due to thalassemia although it is known to occur in polycythemia vera and sickle cell anemia. Cases presenting with paraparesis have been treated with either surgery or radiotherapy with equal frequency and efficacy. Almost all reported cases with paraplegia have been treated with surgery with or without radiation therapy. As paraplegia secondary to $\mathrm{EMH}$ is rare, there is no consensus on treatment. We hereby report a case of thalassemia with paraplegia treated successfully with surgery. Treatment options for cord compression include primarily blood transfusion, surgery, and radiation therapy. Because of the extreme rarity of this condition, direct comparisons between various treatment modalities are not possible. The bias toward surgery is due to its immediate decompressing effect. EMH in thalassemia has also been treated with transfusion therapy with the rationale that correction of anemia would downregulate erythropoietin and lead to reversal of $\mathrm{EMH}$. However, improvement with blood transfusion alone is usually incomplete and slow. Our patient did not improve after adequate blood transfusion and hence underwent surgical decompression after which he showed rapid and complete neurologic recovery. Therefore, we conclude that surgical decompression of EMH tissue has to be considered early in patients presenting with paraplegia if symptom shows no improvement after adequate blood transfusion.
\end{abstract}

\section{Introduction}

Spinal cord compression due to extramedullary hematopoiesis $(\mathrm{EMH})$ is a rare complication of thalassemia and generally presents as paraparesis with sensory impairment. ${ }^{1}$ Complete paraplegia is extremely rare in EMH due to thalassemia although it is known to occur in polycythemia vera and sickle cell anemia. ${ }^{2}$ Cases presenting with paraparesis have been treated with either surgery or radiotherapy ${ }^{3}$ with equal frequency and efficacy. Almost all reported cases with paraplegia have been treated with surgery with or without radiation therapy. As paraplegia secondary to EMH is rare, there is no consensus on treatment. We hereby report a case of thalassemia with paraplegia treated successfully with surgery. received

November 16, 2015 accepted

November 23, 2015

published online

February 19, 2016
DOI http://dx.doi.org/

10.1055/s-0036-1572382. ISSN 2277-954X. (c) 2016 Neurological Surgeons' Society of India

License terms

(c) $(1) \$$ 
B-Thalassemia is due to mutation in the HBB gene on chromosome $11 .^{4}$ inherited in an autosomal-recessive fashion and affecting $\beta$ chain of hemoglobin. EMH is a compensatory phenomenon and commonly involves the liver, spleen, and lymph nodes. ${ }^{3}$ Spinal cord compression due to $\mathrm{EMH}$ is an extremely rare complication. ${ }^{3}$

\section{Case Report}

A 32-year-old man diagnosed to have thalassemia major presented to SJMCH emergency with history of weakness of bilateral lower limbs of 2 months duration with sudden worsening of lower limb power to grade $0 / 5$ for 2 days. He developed acute urinary retention and was catheterized. Through the first decade of life, he maintained a hemoglobin level of 9 to $10 \mathrm{~g} / \mathrm{dL}$ with multiple blood transfusions. He had a similar episode of transient paraparesis in 2011 when his hemoglobin level was $6.8 \mathrm{~g} / \mathrm{dL}$ and patient improved completely after blood transfusion.

His physical examination revealed a height of $158 \mathrm{~cm}$ and weight of $38 \mathrm{~kg}$ with poor muscular development. He had characteristic facies with frontal bossing, prominent malar prominences due to maxillary hypertrophy, and depression of nasal bridge. He was pale and icteric. The liver and spleen were enlarged, 5 and $6 \mathrm{~cm}$ below costal margin respectively. Higher mental functions and cranial nerve examinations were normal. Motor system examination revealed increased tone in lower limb muscles with complete loss of power (grade 0/5) in all muscle groups of both lower limbs. Deep tendon reflexes were exaggerated in both lower limbs with bilateral extensor planter response. There was complete sensory loss below T4 level. Superficial reflexes were absent below T4. His hemoglobin was $6.8 \mathrm{~g} / \mathrm{dL}$ on admission. Magnetic resonance imaging (MRI) of the dorsal spine showed a mass lesion extending from D2 to D10 levels with extradural component causing cord compression. It was isointense on T1-weighted (T1W) images with a high signal intensity rim and hyperintense on T2-weighted (T2W) images. The appearance of the lesion on MRI along with the known underlying hematologic condition was strongly suggestive of EMH (-Fig. 1).

The patient underwent packed red blood cell transfusion with a target to keep his hemoglobin level above $10 \mathrm{~g} / \mathrm{dL}$. He was also started on calcium and folic acid supplements. In spite of adequate correction of hemoglobin with blood transfusion, there was no change in the neurological status. Option of surgical decompression was given. However, patient did not agree for surgery as he was expecting his limb weakness to improve with blood transfusion as in the past. Consent for surgery could finally be obtained when even after 6 days his paraplegia did not improve. He underwent D2-D9 laminectomy and microsurgical decompression of the extradural lesion. Intraoperatively, the posterior spinal elements were hypertrophic and vascular. Fibrous, highly vascular extradural soft tissue component, was found compressing the cord and this was excised. Postoperatively his neurologic status improved significantly. On first follow-up, after 2 weeks, patient's

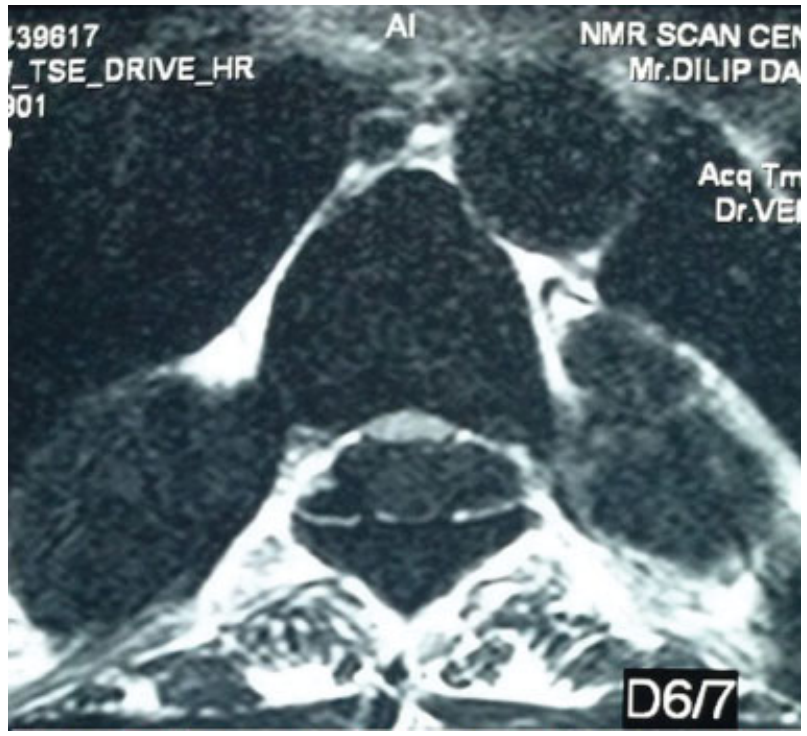

Fig. 1 It is MRI picture taken from a camera with the following description: MRI shows an isointense mass with high signal intensity rim on T1-weighted images and hyperintense images on T2-weighted images.

lower limb power had improved to 5/5 and he had regained bowel and bladder control. Histopathologic examination of the excised tissue showed hypercellular bone marrow due to erythroid hyperplasia (-Fig. 2).

\section{Discussion}

EMH can occur in many disorders, including thalassemia, polycythemia rubra vera, myelofibrosis, hemolytic anemia, and various hemoglobinopathies. ${ }^{3}$ Spinal cord compression due to EMH in thalassemia was first reported by Gatto in $1954 .^{5}$ It usually has a predilection for the lower thoracic spine where the limited mobility and narrow spinal canal predisposes itself

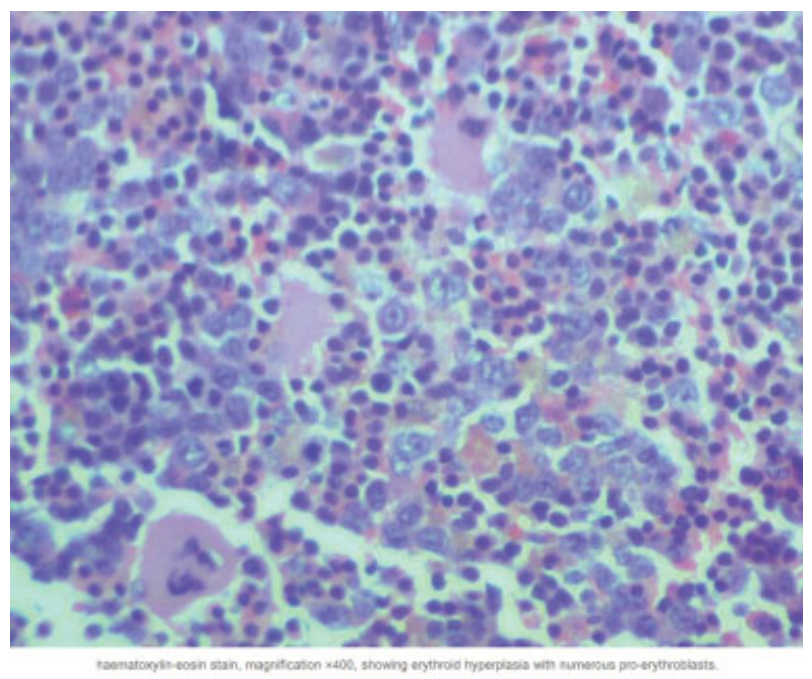

Fig. 2 This picture has been taken from a pathology microscope. Hematoxylin-eosin stain, magnification $x 400$, erythroid hyperplasia with numerous pre-erythroblasts. 
to spinal cord compression ${ }^{6,7}$. Most cases present with paraparesis, sensory impairment, and, occasionally, sphincter disturbances. Complete paraplegia has been reported very rarely in thalassemia and occurs more frequently in polycythemia rubra vera and sickle cell anemia. ${ }^{2}$ The diagnostic procedure of choice is MRI that characteristically shows an isointense mass with a high spinal intensity rim on T1W images and a hyperintense mass onT2W images. ${ }^{8}$ The diagnosis is based on strong clinical suspicion in the presence of diffuse bone marrow hyperplasia along with symmetric paraspinal and epidural masses. Treatment options for cord compression include primarily blood transfusion, surgery, and radiation therapy. Because of the extreme rarity of this condition, direct comparisons between various treatment modalities are not possible.

The bias toward surgery is due to its immediate decompressing effect. EMH in thalassemia has also been treated with transfusion therapy with the rationale that correction of anemia would downregulate erythropoietin and lead to reversal of EMH. However, improvement with blood transfusion alone is usually incomplete and slow. ${ }^{9}$

\section{Conclusion}

Our patient did not improve after adequate blood transfusion and hence underwent surgical decompression after which he showed rapid and complete neurologic recovery. Therefore, we conclude that surgical decompression of EMH tissue has to be considered early in patients presenting with paraplegia if symptom shows no improvement after adequate blood transfusion.

\section{References}

1 Malik M, Pillai LS, Gogia N, et al. Paraplegia due to extramedullary hematopoiesis in thalassemia treated successfully with radiation therapy. J Clin Neurosci 2002;9(6): 721-725

2 Salehi SA, Koski T, Ondra SL. Spinal cord compression in betathalassemia: case report and review of the literature. Spinal Cord 2004;42(2):117-123

3 Malik M, Pillai LS, Gogia N, et al. Paraplegia due to extramedullary hematopoiesis in thalassemia treated successfully with radiation therapy. Haematologica 2007;92(3): e28-e30

4 141900:haemoglobin-beta locus;HBB:http://www.omim.org/ entry $/ 141900$

5 Gatto I, Terrana V, Biondi L. Compression on the spinal proliferation of one marrow in the epidural space in the subject suffering from disease Colley splenectomized. [Italian] Hematological 1954;38:61-75

6 Issaragrisil S, Piankigagum A, Wasi P. Spinal cord compression in thalassemia. Report of 12 cases and recommendations for treatment. Arch Intern Med 1981;141(8):1033-1036

7 Tan TC, Tsao J, Cheung FC. Extramedullary haemopoiesis in thalassemia intermedia presenting as paraplegia. J Clin Neurosci 2002;9(6):721-725

8 Munn RK, Kramer CA, Arnold SM. Spinal cord compression due to extramedullary hematopoiesis in beta-thalassemia intermedia. Int J Radiat Oncol Biol Phys 1998;42(3):607-609

9 Coşkun E, Keskin A, Süzer T, Sermez Y, Kildaci T, Tahta K. Spinal cord compression secondary to extramedullary hematopoiesis in thalassemia intermedia. Eur Spine J 1998;7(6):501-504 